\title{
SINGULAR SUPPORT OF A VERTEX ALGEBRA AND THE ARC SPACE OF ITS ASSOCIATED SCHEME
}

\author{
TOMOYUKI ARAKAWA AND ANDREW R. LINSHAW
}

Dedicated to Professor Anthony Joseph on his seventy-fifth birthday

\begin{abstract}
Attached to a vertex algebra $\mathcal{V}$ are two geometric objects. The associated scheme of $\mathcal{V}$ is the spectrum of Zhu's Poisson algebra $R_{\mathcal{V}}$. The singular support of $\mathcal{V}$ is the spectrum of the associated graded algebra $\operatorname{gr}(\mathcal{V})$ with respect to Li's canonical decreasing filtration. There is a closed embedding from the singular support to the arc space of the associated scheme, which is an isomorphism in many interesting cases. In this note we give an example of a non-quasi-lisse vertex algebra whose associated scheme is reduced, for which the isomorphism is not true as schemes but true as varieties.
\end{abstract}

\section{INTRODUCTION}

Attached to a vertex algebra $\mathcal{V}$ are two geometric objects. The associated scheme $\tilde{X}_{\mathcal{V}}$ of $\mathcal{V}$ is the spectrum of commutative algebra $R_{\mathcal{V}}$, which is an affine Poisson scheme of finite type 1 . The singular support $\operatorname{SS}(\mathcal{V})$ of $\mathcal{V}$ is the spectrum of the associated graded algebra $\operatorname{gr}(\mathcal{V})$ with respect to Li's canonical decreasing filtration, which is a vertex Poisson scheme of infinite type 2 . There is a closed embedding

$$
\Phi: \operatorname{SS}(\mathcal{V}) \hookrightarrow\left(\tilde{X}_{\mathcal{V}}\right)_{\infty}
$$

from the singular support to the arc space $\tilde{X}_{\infty}$ of the associated scheme, which is an isomorphism in many interesting cases.

Originally $\mathrm{Zhu}[\mathrm{Zh}]$ introduced the algebra $R_{\mathcal{V}}$ to define a certain finiteness condition on a vertex algebra. Recall that a vertex algebra $\mathcal{V}$ is called lisse (or $C_{2}$-cofinite) if $\operatorname{dim} \tilde{X}_{\mathcal{V}}=$ 0 . Using the map $\Phi$ one can show that this condition is equivalent to that $\operatorname{dim} \operatorname{SS}(\mathcal{V})=0$, and hence, the lisse condition is a natural finiteness condition ([ArI] $)$. It is known that lisse vertex (operator) algebras have many nice properties, such as modular invariance property of characters $([\mathrm{Zh}, \overline{\mathrm{Mi}}])$, and this condition has been assumed in many significant theories of vertex (operator) algebras. However, recently non-lisse vertex algebras have caught a lot of attention due to the Higgs branch conjecture by Beem and Rastelli [BR], which states that the reduced scheme $X_{\mathcal{V}}$ of $\tilde{X}_{\mathcal{V}}$ should be isomorphic to the Higgs branch of a four-dimensional $N=2$ superconformal field theory $\mathcal{T}$ if $\mathcal{V}$ obtained from $\mathcal{T}$ by the correspondence discovered by [BLL+], see the survey articles [ArII, ArIII] and the references therein.

T. A. is supported by JSPS KAKENHI Grants \#17H01086 and \#17K18724.

A. L. is supported by Simons Foundation Grant \#318755.

We thank Julien Sebag for helpful comments on an earlier draft of this paper.

${ }^{1}$ provided that $\mathcal{V}$ is finitely strongly generated

${ }^{2}$ unless $\mathcal{V}$ is finite-dimensional 
It is natural to ask whether the map $\Phi$ is always an isomorphism, and if not, whether $\Phi$ defines an isomorphism as varieties. Very recently counterexamples to the first question were found by van Ekeren and Heluani $[\mathrm{EH}]$ in the case that $\mathcal{V}$ is lisse in their study of chiral homology of elliptic curves. It was also shown recently in [AMII] that the map $\Phi$ defines an isomorphism as varieties if $\mathcal{V}$ is quasi-lisse, that is, the Poisson variety $X_{\mathcal{V}}$ has finitely many symplectic leaves. In this note we give an example of a non-quasi-lisse vertex algebra whose associated scheme is reduced, for which $\Phi$ is not an isomorphism of schemes, but still defines an isomorphism of varieties. We remark that by tensoring one of the lisse examples in [EH] with any non-quasi-lisse vertex algebra, one can trivially obtain a non-quasi-lisse example. However, all such examples have the property that the associated scheme is nonreduced.

\section{VERTEX ALGEBRAS}

We assume that the reader is familiar with vertex algebras, which have been discussed from various points of view in the literature [B, FLM, K, FBZ]. Given an element $a$ in a vertex algebra $\mathcal{V}$, the field associated to $a$ via the state-field correspondence is denoted by

$$
a(z)=\sum_{n \in \mathbb{Z}} a(n) z^{-n-1} \in \operatorname{End}(\mathcal{V})\left[\left[z, z^{-1}\right]\right]
$$

Throughout this paper, we shall identify $\mathcal{V}$ with the corresponding space of fields. Given $a, b \in \mathcal{V}$, the operators product expansion (OPE) formula is given by

$$
a(z) b(w) \sim \sum_{n \geq 0}\left(a_{(n)} b\right)(w)(z-w)^{-n-1} .
$$

Here $\left(a_{(n)} b\right)(w)=\operatorname{Res}_{z}[a(z), b(w)](z-w)^{n}$ where

$$
[a(z), b(w)]=a(z) b(w)-(-1)^{|a||b|} b(w) a(z),
$$

and $\sim$ means equal modulo terms which are regular at $z=w$. The normally ordered product $: a(z) b(z):$ is defined to be

$$
a(z)_{-} b(z)+(-1)^{|a||b|} b(z) a(z)_{+},
$$

where

$$
a(z)_{-}=\sum_{n<0} a(n) z^{-n-1}, \quad a(z)_{+}=\sum_{n \geq 0} a(n) z^{-n-1} .
$$

We usually omit the formal variable $z$ and write $: a(z) b(z):=: a b:$, when no confusion can arise. For $a_{1}, \ldots, a_{k} \in \mathcal{V}$, the iterated normally ordered product is defined inductively by

$$
: a_{1} a_{2} \cdots a_{k}:=: a_{1}\left(: a_{2} \cdots a_{k}:\right) \text {. }
$$

A subset $S=\left\{a_{i} \mid i \in I\right\}$ of $\mathcal{V}$ is said to strongly generate $\mathcal{V}$, if $\mathcal{V}$ is spanned by the set of normally ordered monomials

$$
: \partial^{k_{1}} a_{i_{1}} \cdots \partial^{k_{m}} a_{i_{m}}:, \quad i_{1}, \ldots, i_{m} \in I, \quad k_{1}, \ldots, k_{m} \geq 0
$$

If $S$ is an ordered strong generating set $\left\{\alpha^{1}, \alpha^{2}, \ldots\right\}$, we say that $S$ freely generates $\mathcal{V}$, if $\mathcal{V}$ has a PBW basis consisting of 


$$
\begin{aligned}
& : \partial^{k_{1}^{1}} \alpha^{i_{1}} \cdots \partial^{k_{r_{1}}^{1}} \alpha^{i_{1}} \partial^{k_{1}^{2}} \alpha^{i_{2}} \cdots \partial^{k_{r_{2}}^{2}} \alpha^{i_{2}} \cdots \partial^{k_{1}^{n}} \alpha^{i_{n}} \cdots \partial^{k_{r_{n}}^{n}} \alpha^{i_{n}}:, \quad 1 \leq i_{1}<\cdots<i_{n}, \\
& k_{1}^{1} \geq k_{2}^{1} \geq \cdots \geq k_{r_{1}}^{1}, \quad k_{1}^{2} \geq k_{2}^{2} \geq \cdots \geq k_{r_{2}}^{2}, \cdots, \quad k_{1}^{n} \geq k_{2}^{n} \geq \cdots \geq k_{r_{n}}^{n}, \\
& k_{1}^{t}>k_{2}^{t}>\cdots>k_{r_{t}}^{t} \text { whenever } \alpha^{i_{t}} \text { is odd. }
\end{aligned}
$$

In particular, the monomials (2.2) are linearly independent, so there are no nontrivial normally ordered polynomial relations among the generators and their derivatives.

$\beta \gamma$-system. The $\beta \gamma$-system $\mathcal{S}$ is freely generated by even fields $\beta, \gamma$ satisfying

$$
\begin{aligned}
\beta(z) \gamma(w) & \sim(z-w)^{-1}, & \gamma(z) \beta(w) & \sim-(z-w)^{-1}, \\
\beta(z) \beta(w) & \sim 0, & \gamma(z) \gamma(w) & \sim 0 .
\end{aligned}
$$

It has Virasoro element $L^{\mathcal{S}}=\frac{1}{2}(: \beta \partial \gamma:-: \partial \beta \gamma:)$ of central charge $c=-1$, under which $\beta, \gamma$ are primary of weight $\frac{1}{2}$.

$\mathcal{W}_{3}$-algebra. The $\mathcal{W}_{3}$-algebra $\mathcal{W}_{3}^{c}$ with central charge $c$ was introduced by Zamolodchikov [Za]. It is an extension of the Virasoro algebra, and is freely generated by a Virasoro field $L$ and an even weight 3 primary field $W$. In fact, $\mathcal{W}_{3}^{c}$ is isomorphic to the principal $\mathcal{W}$ algebra $\mathcal{W}^{k}\left(\mathfrak{s l}_{3}, f_{\text {prin }}\right)$ where $c=2-\frac{24(k+2)^{2}}{k+3}$. For generic values of $c, \mathcal{W}_{3}^{c}$ is simple, but for certain special values it has a nontrivial ideal. In this paper, we only need the case $c=-2$, which is nongeneric. We shall denote the simple graded quotient of $\mathcal{W}_{3}^{-2}$ by $\mathcal{W}$ for the rest of the paper. Since $\mathcal{W}_{3}^{-2}$ has a nontrivial ideal, $\mathcal{W}$ is strongly but not freely generated by $L, W$.

There is a useful embedding $i: \mathcal{W} \rightarrow \mathcal{S}$ due to Wang [WaI], given by

$$
\begin{aligned}
& L \mapsto \frac{1}{2}: \beta \beta \gamma \gamma:+: \beta(\partial \gamma):-:(\partial \beta) \gamma: \\
& \left.W \mapsto \frac{1}{4 \sqrt{2}}\left(2: \beta^{3} \gamma^{3}:+9: \beta^{2}(\partial \gamma) \gamma:+3: \beta \partial^{2} \gamma:-9:(\partial \beta) \beta \gamma^{2}:-12 \partial \beta\right)(\partial \gamma):+3:\left(\partial^{2} \beta\right) \gamma:\right),
\end{aligned}
$$

and we shall identify $\mathcal{W}$ with its image in $\mathcal{S}$. In fact, $\mathcal{W}$ is precisely the subalgebra of $\mathcal{S}$ that commutes with the Heisenberg algebra generated by : $\beta \gamma:$. Note that $W$ is normalized so that it satisfies

$$
\begin{gathered}
W(z) W(w) \sim-\frac{9}{8}(z-w)^{-6}+\frac{27}{8} L(w)(z-w)^{-4}+\frac{27}{16} \partial L(w)(z-w)^{-3} \\
+\left(\frac{9}{2}: L L:-\frac{27}{32} \partial^{2} L\right)(w)(z-w)^{-2}+\left(\frac{9}{2}:(\partial L) L:-\frac{3}{16} \partial^{3} L\right)(w)(z-w)^{-1} .
\end{gathered}
$$

This normalization is nonstandard but convenient for our purposes.

Zhu's commutative algebra and the associated variety. Given a vertex algebra $\mathcal{V}$, define

$$
C(\mathcal{V})=\operatorname{Span}\left\{a_{(-2)} b \mid a, b \in \mathcal{V}\right\}, \quad R_{\mathcal{V}}=\mathcal{V} / C(\mathcal{V}) .
$$

It is well known that $R_{\mathcal{V}}$ is a commutative, associative algebra with product induced by the normally ordered product $[\overline{Z h}]$. Also, if $\mathcal{V}$ is graded by conformal weight, $R_{\mathcal{V}}$ inherits this grading. Define the associated scheme

$$
\tilde{X}_{\mathcal{V}}=\operatorname{Spec}\left(R_{\mathcal{V}}\right)
$$


and the associated variety

$$
X_{\mathcal{V}}=\operatorname{Specm}\left(R_{\mathcal{V}}\right)=\left(\tilde{X}_{\mathcal{V}}\right)_{\text {red }}
$$

Here $\left(\tilde{X}_{\mathcal{V}}\right)_{\text {red }}$ denotes the reduced scheme of $\tilde{X}_{\mathcal{V}}$. If $\left\{\alpha_{i} \mid i \in I\right\}$ is a strong generating set for $\mathcal{V}$, the images of these fields in $R_{\mathcal{V}}$ will generate $R_{\mathcal{V}}$ as a ring. In particular, $R_{\mathcal{V}}$ is finitely generated if and only if $\mathcal{V}$ is strongly finitely generated.

Since the $\beta \gamma$-system $\mathcal{S}$ is freely generated by $\beta, \gamma, R_{\mathcal{S}} \cong \mathbb{C}[b, g]$, where $b, g$ denote the images of $\beta, \gamma$ in $R_{\mathcal{S}}$. On the other hand, since $\mathcal{W}$ is not freely generated by $L, W$, the structure of $R_{\mathcal{W}}$ is more complicated.

Lemma 2.1. Let $\ell, w$ denote the images of $L, W$ in $R_{\mathcal{W}}$. Then $R_{\mathcal{W}} \cong \mathbb{C}[\ell, w] /\left\langle w^{2}-\ell^{3}\right\rangle$.

Proof. Since $\mathcal{W}$ is strongly generated by $L, W, R_{\mathcal{W}}$ is generated by $\ell, w$, so $R_{\mathcal{W}} \cong \mathbb{C}[\ell, w] / I$ for some ideal $I$. By Lemma 2.1 of [WaII], we have the following normally ordered relation in $\mathcal{W}$ at weight 6 :

$$
: W^{2}:-: L^{3}:-\frac{7}{8}:\left(\partial^{2} L\right) L:-\frac{19}{32}:(\partial L)^{2}:=0 .
$$

Note that (2.8) differs slightly from the formula in [WaII] because our normalization of $W$ is different. It follows that $w^{2}-\ell^{3} \in I$.

To see that $I \subseteq\left\langle w^{2}-\ell^{3}\right\rangle$, let $p=p(\ell, w) \in I$. Without loss of generality, we may assume $p$ is homogeneous of weight $d$. It must come from a normally ordered polynomial relation

$$
P=P(L, \partial L, \ldots, W, \partial W, \ldots)=0
$$

of weight $d$ in $\mathcal{W}$ among $L, W$ and their derivatives. The monomials of $p$ correspond to the normally ordered monomials of $P$ which do not lie in $C(\mathcal{W})$, and have the form

$$
: L^{i} W^{j}:, \quad 2 i+3 j=d .
$$

Using (2.8) repeatedly, we can rewrite this relation in the form

$$
P^{\prime}=P^{\prime}(L, \partial L, \ldots, W, \partial W, \ldots)=0,
$$

where all terms of the form (2.9) that appear either have $j=0$ or $j=1$. In fact, since $P^{\prime}$ is homogeneous of weight $d$, we must have $j=0$ if $d$ is even, and $j=1$ if $d$ is odd, so only one such term can appear. If this term appears with nonzero coefficient, as a normally ordered polynomial in $\beta, \gamma$ and their derivatives, it will contribute the term : $\beta^{2 i+3 j} \gamma^{2 i+3 j}$ :, which cannot be canceled. This contradicts $P^{\prime}=0$, so each monomial in $P^{\prime}$ must lie in $C(\mathcal{W})$. Equivalently, $p \in\left\langle w^{2}-\ell^{3}\right\rangle$.

\section{JET SCHEMES AND ARC SPACES}

We recall some basic facts about jet schemes, following the notation in [EM]. Let $X$ be an irreducible scheme over $\mathbb{C}$ of finite type. The first jet scheme $X_{1}$ is the total tangent space of $X$, and for $m>1$ the jet schemes $X_{m}$ are higher-order generalizations which are determined by their functor of points. Given a $\mathbb{C}$-algebra $A$, we have a bijection

$$
\operatorname{Hom}\left(\operatorname{Spec}(A), X_{m}\right) \cong \operatorname{Hom}\left(\operatorname{Spec}\left(A[t] /\left\langle t^{m+1}\right\rangle\right), X\right) \text {. }
$$

Thus the $\mathbb{C}$-valued points of $X_{m}$ correspond to the $\mathbb{C}[t] /\left\langle t^{m+1}\right\rangle$-valued points of $X$. For $p>m$, we have projections $\pi_{p, m}: X_{p} \rightarrow X_{m}$ and $\pi_{p, m} \circ \pi_{q, p}=\pi_{q, m}$ when $q>p>m$. The assignment $X \mapsto X_{m}$ is functorial, and a morphism $f: X \rightarrow Y$ induces $f_{m}: X_{m} \rightarrow Y_{m}$ 
for all $m \geq 1$. If $X$ is nonsingular, $X_{m}$ is irreducible and nonsingular for all $m$. If $X, Y$ are nonsingular and $f: X \rightarrow Y$ is a smooth surjection, $f_{m}$ is surjective for all $m$.

For an affine scheme $X=\operatorname{Spec}(R)$ where $R=\mathbb{C}\left[y_{1}, \ldots, y_{r}\right] /\left\langle f_{1}, \ldots, f_{k}\right\rangle, X_{m}$ is also affine and we can give explicit equations for $X_{m}$ as follows. Define variables $y_{1}^{(i)}, \ldots y_{r}^{(i)}$ for $i=0, \ldots, m$, and define a derivation $D$ by

$$
D\left(y_{j}^{(i)}\right)=\left\{\begin{array}{cc}
y_{j}^{(i+1)} & 0 \leq i<m \\
0 & i=m
\end{array}\right.
$$

which specifies its action on all of $\mathbb{C}\left[y_{1}^{(i)}, \ldots, y_{r}^{(i)}\right]$, for $0 \leq i \leq m$. In particular, $f_{\ell}^{(i)}=D^{i}\left(f_{\ell}\right)$ is a well-defined polynomial in $\mathbb{C}\left[y_{1}^{(i)}, \ldots, y_{r}^{(i)}\right]$. Letting

$$
R_{m}=\mathbb{C}\left[y_{1}^{(i)}, \ldots, y_{r}^{(i)}\right] /\left\langle f_{1}^{(i)}, \ldots, f_{k}^{(i)}\right\rangle,
$$

we have $X_{m} \cong \operatorname{Spec}\left(R_{m}\right)$. By identifying $y_{j}$ with $y_{j}^{(0)}$, we may identify $R$ with a subalgebra of $R_{m}$. There is a $\mathbb{Z}_{\geq 0}$-grading on $R_{m}$ which we call height, given by

$$
R_{m}=\bigoplus_{n \geq 0} R_{m}[n], \quad \operatorname{ht}\left(y_{j}^{(i)}\right)=i .
$$

For all $m, R_{m}[0]=R$ and $R_{m}[n]$ is an $R$-module.

Given a scheme $X$, define

$$
X_{\infty}=\lim _{\leftarrow} X_{m}
$$

which is known as the arc space of $X$. For a $\mathbb{C}$-algebra $A$, we have a bijection

$$
\operatorname{Hom}\left(\operatorname{Spec}(A), X_{\infty}\right) \cong \operatorname{Hom}(\operatorname{Spec}(A[[t]]), X),
$$

so the $\mathbb{C}$-valued points of $X_{\infty}$ correspond to the $\mathbb{C}[[t]]$-valued points of $X$. If $X=\operatorname{Spec}(R)$ as above,

$$
X_{\infty} \cong \operatorname{Spec}\left(R_{\infty}\right), \text { where } R_{\infty}=\mathbb{C}\left[y_{1}^{(i)}, \ldots, y_{r}^{(i)}\right] /\left\langle f_{1}^{(i)}, \ldots, f_{k}^{(i)}\right\rangle .
$$

Here $i \geq 0$, and $D\left(y_{j}^{(i)}\right)=y_{j}^{(i+1)}$ for all $i$.

By a theorem of Kolchin [Kol], $X_{\infty}$ is irreducible if $X$ is irreducible. However, even if $X$ is irreducible and reduced, $X_{\infty}$ need not be reduced. The following result is due to Sebag (see Example 8 of [SI], as well as more general results in [SII]), but we include a proof for the benefit of the reader.

Lemma 3.1. For $X=\operatorname{Spec}\left(\mathbb{C}[\ell, w] /\left\langle w^{2}-\ell^{3}\right\rangle\right)=\tilde{X}_{\mathcal{W}}, X_{\infty}$ is not reduced.

Proof. We have

$$
X_{\infty} \cong \operatorname{Spec}\left(R_{\infty}\right), \quad R_{\infty}=\mathbb{C}\left[\ell^{(0)}, \ell^{(1)}, \ldots, w^{(0)}, w^{(1)}, \ldots\right] /\left\langle f^{(0)}, f^{(1)}, \ldots\right\rangle,
$$

where $f^{(0)}=\left(\ell^{(0)}\right)^{3}-\left(w^{(0)}\right)^{2}$. Consider the element

$$
r_{1}=3 \ell^{(1)} w^{(0)}-2 \ell^{(0)} w^{(1)} \in \mathbb{C}\left[\ell^{(0)}, \ell^{(1)}, \ldots, w^{(0)}, w^{(1)}, \ldots\right] .
$$


First, $r_{1} \notin\left\langle f^{(0)}, f^{(1)}, \ldots\right\rangle$ since no element of this ideal has leading term of degree 2 . However, $\left(r_{1}\right)^{3} \in\left\langle f^{(0)}, f^{(1)}, \ldots\right\rangle$; a calculation shows that

$$
\begin{aligned}
\left(r_{1}\right)^{3} & =\left(-81 \ell^{(0)} \ell^{(1)} \ell^{(2)} w^{(0)}-\frac{27}{2}\left(\ell^{(0)}\right)^{2} \ell^{(3)} w^{(0)}+18\left(\ell^{(0)}\right)^{2} \ell^{(2)} w^{(1)}-4\left(w^{(1)}\right)^{3}+15 w^{(0)} w^{(1)} w^{(2)}\right. \\
& \left.+9\left(w^{(0)}\right)^{2} w^{(3)}\right) f^{(0)}+\left(\frac{9}{2}\left(\ell^{(0)}\right)^{2} \ell^{(2)} w^{(0)}+12\left(\ell^{(0)}\right)^{2} \ell^{(1)} w^{(1)}-7 w^{(0)}\left(w^{(1)}\right)^{2}-3\left(w^{(0)}\right)^{2} w^{(2)}\right) f^{(1)} \\
& +\left(-\frac{9}{2}\left(\ell^{(0)}\right)^{2} \ell^{(1)} w^{(0)}-6\left(\ell^{(0)}\right)^{3} w^{(1)}+9\left(w^{(0)}\right)^{2} w^{(1)}\right) f^{(2)}+\left(\frac{9}{2}\left(\ell^{(0)}\right)^{3} w^{(0)}-\frac{9}{2}\left(w^{(0)}\right)^{3}\right) f^{(3)} .
\end{aligned}
$$

Therefore regarded as an element of $R_{\infty}, r_{1} \neq 0$ but $\left(r_{1}\right)^{3}=0$.

It is well known that in characteristic zero, for any affine scheme $X$, the nilradical $\mathcal{N} \subseteq$ $\mathcal{O}\left(X_{\infty}\right)$ is a differential ideal; in other words, $D(\mathcal{N}) \subseteq \mathcal{N}$. A natural question (see [KS]) is whether $\mathcal{N}$ is finitely generated as a differential ideal, and whether an explicit generating set can be found. In general, $\mathcal{N}$ need not be finitely generated; this was shown for $X=$ $\operatorname{Spec}(\mathbb{C}[x, y] /\langle x y\rangle)$ in $[\mathrm{BS}]$. In the example $X=\operatorname{Spec}\left(\mathbb{C}[\ell, w] /\left\langle w^{2}-\ell^{3}\right\rangle\right)$, a calculation shows that in addition to $r_{1}$,

$$
r_{2}=\left(w^{(1)}\right)^{2}-\frac{9}{4} \ell^{(0)}\left(\ell^{(1)}\right)^{2}
$$

does not lie in $\left\langle f^{(0)}, f^{(1)}, \ldots\right\rangle$, but $\left(r_{2}\right)^{3}$ does. So $r_{2}$ is another nontrivial element of $\mathcal{N}$. We expect that $\mathcal{N}$ is generated as a differential ideal by $r_{1}$ and $r_{2}$.

The following characterization of $\mathcal{N}$ in this example will also be useful to us.

Lemma 3.2. Let $X=\operatorname{Spec}\left(\mathbb{C}[\ell, w] /\left\langle w^{2}-\ell^{3}\right\rangle\right)$ and let $t$ be a coordinate function on $\mathbb{C}$. Consider the map

$$
\mathbb{C} \rightarrow X, \quad t \mapsto\left(t^{2}, t^{3}\right)
$$

and the induced homomorphism

$$
\varphi: \mathcal{O}\left(X_{\infty}\right) \rightarrow \mathcal{O}\left(\mathbb{C}_{\infty}\right), \quad \ell^{(0)} \mapsto\left(t^{(0)}\right)^{2}, \quad w^{(0)} \mapsto\left(t^{(0)}\right)^{3} .
$$

Then $\mathcal{N}=\operatorname{ker}(\varphi)$.

Proof. Since (3.8) is birational, the map $\mathbb{C}_{\infty} \rightarrow X_{\infty}$ on arc spaces induced by (3.8) is dominant, see Proposition 3.2 of $[\overline{E M}]$. Therefore $\operatorname{ker}(\varphi) \subseteq \mathcal{N}$. On the other hand, $\mathcal{N} \subseteq \operatorname{ker}(\varphi)$ since $\mathcal{O}\left(\mathbb{C}_{\infty}\right) \cong \mathbb{C}\left[t^{(0)}, t^{(1)}, \ldots\right]$, which is an integral domain.

\section{LI'S FILTRATION AND SINGULAR SUPPORT}

For any vertex algebra $\mathcal{V}$, we have Li's canonical decreasing filtration

$$
F^{0}(\mathcal{V}) \supseteq F^{1}(\mathcal{V}) \supseteq \cdots
$$

where $F^{p}(\mathcal{V})$ is spanned by elements of the form

$$
\text { : } \partial^{n_{1}} a^{1} \partial^{n_{2}} a^{2} \cdots \partial^{n_{r}} a^{r}:,
$$


where $a^{1}, \ldots, a^{r} \in \mathcal{V}, n_{i} \geq 0$, and $n_{1}+\cdots+n_{r} \geq p[\overline{\mathrm{LiI}}]$. Clearly $\mathcal{V}=F^{0}(\mathcal{V})$ and $\partial F^{i}(\mathcal{V}) \subseteq$ $F^{i+1}(\mathcal{V})$. Set

$$
\operatorname{gr}(\mathcal{V})=\bigoplus_{p \geq 0} F^{p}(\mathcal{V}) / F^{p+1}(\mathcal{V})
$$

and for $p \geq 0$ let

$$
\sigma_{p}: F^{p}(\mathcal{V}) \rightarrow F^{p}(\mathcal{V}) / F^{p+1}(\mathcal{V}) \subseteq \operatorname{gr}(\mathcal{V})
$$

be the projection. Note that $\operatorname{gr}(\mathcal{V})$ is a graded commutative algebra with product

$$
\sigma_{p}(a) \sigma_{q}(b)=\sigma_{p+q}\left(a_{(-1)} b\right)
$$

for $a \in F^{p}(\mathcal{V})$ and $b \in F^{q}(\mathcal{V})$. We say that the subspace $F^{p}(\mathcal{V}) / F^{p+1}(\mathcal{V})$ has height $p$. Note that $\operatorname{gr}(\mathcal{V})$ has a differential $\partial$ defined by

$$
\partial\left(\sigma_{p}(a)\right)=\sigma_{p+1}(\partial a)
$$

for $a \in F^{p}(\mathcal{V})$. Finally, $\operatorname{gr}(\mathcal{V})$ has the structure of a Poisson vertex algebra [LiI]; for $n \geq 0$, we define

$$
\sigma_{p}(a)_{(n)} \sigma_{q}(b)=\sigma_{p+q-n} a_{(n)} b .
$$

Zhu's commutative algebra $R_{\mathcal{V}}$ is isomorphic to the subalgebra $F^{0}(\mathcal{V}) / F^{1}(\mathcal{V}) \subseteq \operatorname{gr}(\mathcal{V})$, since $F^{1}(\mathcal{V})$ coincides with the space $C(\mathcal{V})$ defined by (2.5). Moreover, $\operatorname{gr}(\mathcal{V})$ is generated by $R_{\mathcal{V}}$ as a differential graded commutative algebra [LiI]. Since $\tilde{X}_{\mathcal{V}}=\operatorname{Spec}\left(R_{\mathcal{V}}\right)$, there is always a surjective homomorphism of differential graded rings

$$
\Phi_{\mathcal{V}}: \mathcal{O}\left(\left(\tilde{X}_{\mathcal{V}}\right)_{\infty}\right) \rightarrow \operatorname{gr}(\mathcal{V})
$$

where the grading on $\mathcal{O}\left(\left(\tilde{X}_{\mathcal{V}}\right)_{\infty}\right)$ is given by (3.2). Define the singular support

$$
\mathrm{SS}(\mathcal{V})=\operatorname{Spec}(\operatorname{gr}(\mathcal{V}))
$$

which is then a subscheme of $\left(\tilde{X}_{\mathcal{V}}\right)_{\infty}$. A natural question which was raised by Arakawa and Moreau [AMI] is whether the map (4.1) is always an isomorphism. This is true in many examples and it was recently shown in [AMII] to hold as varieties when $\mathcal{V}$ is quasilisse, that is, if $X_{\mathcal{V}}$ has finitely many symplectic leaves, see [AK] for the details. We note that the vertex algebra $\mathcal{W}$ is not quasi-lisse.

\section{MAIN RESULT}

Theorem 5.1. For the vertex algebra $\mathcal{W}$, the map $\Phi_{\mathcal{W}}: \mathcal{O}\left(\left(\tilde{X}_{\mathcal{W}}\right)_{\infty}\right) \rightarrow g r(\mathcal{W})$ is not injective, so $\left(\tilde{X}_{\mathcal{W}}\right)_{\infty}$ and $S S(\mathcal{W})$ are not isomorphic as schemes.

Proof. As before, we use the notation

$$
\mathcal{O}\left(\left(\tilde{X}_{\mathcal{W}}\right)_{\infty}\right) \cong R_{\infty}=\mathbb{C}\left[\ell^{(0)}, \ell^{(1)}, \ldots, w^{(0)}, w^{(1)}, \ldots\right] /\left\langle f^{(0)}, f^{(1)}, \ldots\right\rangle .
$$

We use the same notation $\partial^{i} L, \partial^{i} W$ to denote the images of the fields $\partial^{i} L, \partial^{i} W \in \mathcal{W}$ in the subspace $F^{i}(\mathcal{W}) / F^{i+1}(\mathcal{W})$ of $\operatorname{gr}(\mathcal{W})$. We therefore may identify $\operatorname{gr}(\mathcal{W})$ with a quotient of the polynomial ring $\mathbb{C}[L, \partial L, \ldots, W, \partial W, \ldots]$. In this notation, $\Phi_{\mathcal{W}}\left(\ell^{(0)}\right)=L$ and $\Phi_{\mathcal{W}}\left(w^{(0)}\right)=W$.

We will show that the nilpotent elements $r_{1}$ and $r_{2}$ in $\mathcal{O}\left(\left(\tilde{X}_{\mathcal{W}}\right)_{\infty}\right)$ given by (3.5) and (3.7), lie in $\operatorname{ker}\left(\Phi_{\mathcal{W}}\right)$. By Lemma 2.1 of [WaII], we have the following relation in $\mathcal{W}$ at weight 6 :

$$
3:(\partial L) W:-2: L(\partial W):+\frac{1}{4} \partial^{3} W=0 .
$$


Therefore in $F^{1}(\mathcal{W}) / F^{2}(\mathcal{W})$, we have the relation

$$
3(\partial L) W-2 L \partial W=0 .
$$

Since $\Phi_{\mathcal{W}}\left(r_{1}\right)=3(\partial L) W-2 L \partial W, r_{1} \in \operatorname{ker}\left(\Phi_{\mathcal{W}}\right)$.

Similarly, in $\mathcal{W}$ we have the following relation in weight 8 :

$$
:(\partial W)^{2}:-\frac{9}{4}:(\partial L)^{2} L:-\frac{3}{16}:\left(\partial^{4} L\right) L:-\frac{3}{8}:\left(\partial^{3} L\right)(\partial L):-\frac{9}{32}:\left(\partial^{2} L\right)^{2}:+\frac{1}{160} \partial^{6} L=0,
$$

so in $F^{2}(\mathcal{W}) / F^{3}(\mathcal{W})$ we have the relation $(\partial W)^{2}-\frac{9}{4}(\partial L)^{2} L=0$, and $r_{2} \in \operatorname{ker}\left(\Phi_{\mathcal{W}}\right)$.

Theorem 5.2. Even though $\left(\tilde{X}_{\mathcal{W}}\right)_{\infty}$ and $S S(\mathcal{W})$ differ as schemes, the map of varieties

$$
S S(\mathcal{W})_{\text {red }} \rightarrow\left(\left(\tilde{X}_{\mathcal{W}}\right)_{\infty}\right)_{\text {red }}
$$

induced by $\Phi_{\mathcal{W}}$, is an isomorphism.

Proof. It suffices to show that the map $\varphi: \mathcal{O}\left(\left(\tilde{X}_{\mathcal{W}}\right)_{\infty}\right) \rightarrow \mathcal{O}\left(\mathbb{C}_{\infty}\right)$ given by (3.9) factors through the map $\Phi_{\mathcal{W}}: \mathcal{O}\left(\left(\tilde{X}_{\mathcal{W}}\right)_{\infty}\right) \rightarrow \operatorname{gr}(\mathcal{W})$, since $\operatorname{ker}(\varphi)=\mathcal{N}$. First, the embedding $i: \mathcal{W} \rightarrow \mathcal{S}$ given by (2.4) induces a map

$$
\operatorname{gr}(i): \operatorname{gr}(\mathcal{W}) \rightarrow \operatorname{gr}(\mathcal{S})
$$

Identifying $\operatorname{gr}(\mathcal{S})$ with $\mathbb{C}[\beta, \partial \beta, \ldots, \gamma, \partial \gamma, \ldots]$, this map is given on generators by

$$
\operatorname{gr}(i)(L)=(\beta \gamma)^{2}, \quad \operatorname{gr}(i)(W)=(\beta \gamma)^{3} .
$$

We also have an injective map of differential graded algebras

$$
\psi: \mathcal{O}\left(\mathbb{C}_{\infty}\right) \rightarrow \operatorname{gr}(\mathcal{S})
$$

defined on the generator $t^{(0)}$ of $\mathcal{O}\left(\mathbb{C}_{\infty}\right)$ by $\psi\left(t^{(0)}\right)=\beta \gamma$. Since

$$
\Phi_{\mathcal{W}}\left(\ell^{(0)}\right)=(\beta \gamma)^{2}=\operatorname{gr}(i)(L)=\psi\left(\left(t^{(0)}\right)^{2}\right), \quad \Phi_{\mathcal{W}}\left(w^{(0)}\right)=(\beta \gamma)^{3}=\operatorname{gr}(i)(W)=\psi\left(\left(t^{(0)}\right)^{3}\right),
$$

and $L, W$ generate $\operatorname{gr}(\mathcal{W})$ as a differential algebra, it is clear that

$$
\operatorname{gr}(i)(\operatorname{gr}(\mathcal{W}))=\psi(A) \cong A=\varphi\left(\mathcal{O}\left(\left(\tilde{X}_{\mathcal{W}}\right)_{\infty}\right)\right),
$$

where $A \subseteq \mathcal{O}\left(\mathbb{C}_{\infty}\right)$ is the subalgebra generated by $\left(t^{(0)}\right)^{2},\left(t^{(0)}\right)^{3}$, and their derivatives. This completes the proof.

In this example, we expect that $\operatorname{gr}(i): \operatorname{gr}(\mathcal{W}) \rightarrow \operatorname{gr}(\mathcal{S})$ is injective, so that $\operatorname{gr}(\mathcal{W}) \cong A$, and in particular is reduced. However, we caution the reader that the associated graded functor is not left exact in general.

\section{FAILURE OF ASSOCIATED GRADED FUNCTOR TO BE LEFT EXACT}

Here we give an example of a simple vertex algebra $\mathcal{V}$ which has a free field realization $i: \mathcal{V} \rightarrow \mathcal{H}$ where $\mathcal{H}$ is the Heisenberg algebra, such that the induced map $\operatorname{gr}(i): \operatorname{gr}(\mathcal{V}) \rightarrow$ $\operatorname{gr}(\mathcal{H})$ is not injective.

First, $\mathcal{H}$ is generated by an even field $\alpha$ satisfying

$$
\alpha(z) \alpha(w) \sim(z-w)^{-2},
$$

and has Virasoro element $L=\frac{1}{2}: \alpha \alpha$ : of central charge $c=1$. There is an action of $\mathbb{Z}_{2}$ sending $\alpha \mapsto-\alpha$ which preserves $L$, and we consider the orbifold

$$
\mathcal{V}=\mathcal{H}^{\mathbb{Z}_{2}} \text {. }
$$


By a result of Dong and Nagatomo $[\overline{\mathrm{DN}}], \mathcal{V}$ is strongly generated by $L$ together with a unique up to scalar weight 4 field primary field

$$
W^{4}=-\frac{1}{6 \sqrt{6}}: \alpha^{4}:-\frac{1}{4 \sqrt{6}}:(\partial \alpha)^{2}:+\frac{1}{6 \sqrt{6}}:\left(\partial^{2} \alpha\right) \alpha:
$$

which is normalized so that it satisfies

$$
W^{4}(z) W^{4}(w) \sim \frac{1}{4}(z-w)^{-8}+\cdots .
$$

One can check by direct calculation that $\mathcal{V}$ is isomorphic to the simple, principal $\mathcal{W}$ algebra of $\mathfrak{s p}_{4}$ with central charge $c=1$. It is convenient to replace $W^{4}$ with the field

$$
W=\frac{35}{132}:\left(\partial^{2} \alpha\right) \alpha:=\frac{35 \sqrt{2 / 3}}{33} W^{4}+\frac{70}{297}: L^{2}:+\frac{35}{396} \partial^{2} L,
$$

which is not primary. A calculation shows that we have the following nontrivial relations in $\mathcal{V}$ at weights 8 and 10 , respectively.

$$
\begin{gathered}
: W^{2}:-: L^{2} W:+\frac{35}{132}:\left(\partial^{2} L\right) L^{2}:-\frac{35}{264}:(\partial L)^{2} L:+\frac{13265}{69696}:\left(\partial^{4} L\right) L: \\
+\frac{19495}{139392}:\left(\partial^{3} L\right) \partial L:-\frac{59}{88}:\left(\partial^{2} L\right) W:-\frac{497}{352}:(\partial L) \partial W:-\frac{181}{528}: L \partial^{2} W: \\
-\frac{139}{2112} \partial^{4} W+\frac{10955}{557568} \partial^{6} L=0, \\
: L^{3} W:+\frac{4455}{1024}:(\partial W) \partial W:-\frac{35}{132}:\left(\partial^{2} L\right) L^{3}:+\frac{35}{264}:(\partial L)^{2} L^{2}:+\frac{347}{256}:(\partial L)^{2} W:- \\
\frac{1069}{256}:(\partial L) L \partial W:-\frac{49}{16}: L^{2} \partial^{2} W:+\frac{385}{576}:\left(\partial^{4} L\right) L^{2}:+\frac{48965}{101376}:\left(\partial^{3} L\right)(\partial L) L:- \\
\frac{35}{44}:\left(\partial^{2} L\right)^{2} L:+\frac{35}{88}:\left(\partial^{2} L\right)(\partial L)^{2}:-\frac{1687}{1536}:\left(\partial^{4} L\right) W:-\frac{5939}{3072}:\left(\partial^{3} L\right)(\partial W):- \\
\frac{247}{256}:\left(\partial^{2} L\right)\left(\partial^{2} W\right):-\frac{10927}{6144}:(\partial L)\left(\partial^{3} W\right):+\frac{779}{1536}: L \partial^{4} W:+\frac{3899}{36864}:\left(\partial^{6} L\right) L:+ \\
\frac{102851}{270336}:\left(\partial^{5} L\right) \partial L:+\frac{7525}{67584}:\left(\partial^{4} L\right) \partial^{2} L:+\frac{659645}{4866048}:\left(\partial^{3} L\right)^{2}: \\
+\frac{68311}{6488064} \partial^{8} L-\frac{3187}{49152} \partial^{6} W=0 .
\end{gathered}
$$

Lemma 6.1. Let $\ell, w$ denote the images of $L, W$ in $R_{\mathcal{V}}$. Then

$$
R_{\mathcal{V}} \cong \mathbb{C}[\ell, w] / I
$$

where I is the ideal generated by $w\left(w-\ell^{2}\right)$ and $\ell^{3} w$. In particular, $\tilde{X}_{\mathcal{V}}=\operatorname{Spec}\left(R_{\mathcal{V}}\right)$ is irreducible of dimension one, but is not reduced.

Proof. Since $\mathcal{V}$ is strongly generated by $L, W$, it follows from (6.1) and (6.2) that $R_{\mathcal{V}} \cong$ $\mathbb{C}[\ell, w] / I$ for some ideal $I$ which contains $w\left(w-\ell^{2}\right)$ and $\ell^{3} w$. The proof that $I$ is generated by these two elements is similar to the proof of Lemma 2.1, and is omitted. Since $I$ is contained in the ideal $\langle w\rangle$, the map $\mathbb{C}[\ell] \rightarrow R_{\mathcal{V}}$ is injective, and $R_{\mathcal{V}}$ has Krull dimension 1. Since $w$ is a nontrivial nilpotent element of $R_{\mathcal{V}}, \tilde{X}_{\mathcal{V}}$ is not reduced. Finally, it is easy to see that the nilradical $\mathcal{N}$ of $R_{\mathcal{V}}$ is generated by $w$, so $\mathcal{N}$ is prime and $\tilde{X}_{\mathcal{V}}$ is irreducible. 
Corollary 6.2. Let $i: \mathcal{V} \rightarrow \mathcal{H}$ be the inclusion. Since $\operatorname{gr}(\mathcal{H})$ is the polynomial ring $\mathbb{C}[\alpha, \partial \alpha, \ldots]$, the induced map $g r(i): \operatorname{gr}(\mathcal{V}) \rightarrow \operatorname{gr}(\mathcal{H})$ is not injective.

In fact, it is easy to verify that the image of $\operatorname{gr}(i)$ is just the differential polynomial algebra generated by $\operatorname{gr}(i)(L)=\frac{1}{2} \alpha^{2}$. Finally, we remark that as in our main example $\mathcal{W}$, the map $\Phi_{\mathcal{V}}: \mathcal{O}\left(\left(\tilde{X}_{\mathcal{V}}\right)_{\infty}\right) \rightarrow \operatorname{gr}(\mathcal{V})$ is not injective for $\mathcal{V}=\mathcal{H}^{\mathbb{Z}_{2}}$. For example,

$$
r=\ell^{(0)} \ell^{(2)} w^{(0)}+\left(\ell^{(1)}\right)^{2} w^{(0)}-\frac{1}{2}\left(\ell^{(0)}\right)^{2} w^{(2)}
$$

is a nontrivial element of $\operatorname{ker}\left(\Phi_{\mathcal{V}}\right)$. In fact, $r$ is nilpotent in $\mathcal{O}\left(\left(\tilde{X}_{\mathcal{V}}\right)_{\infty}\right)$ and satisfies $r^{3}=0$.

\section{UNIVERSAL ENVELOPING VERTEX ALGEBRAS}

Let $\mathcal{V}$ be a conformal vertex algebra with a strong generating set $S$, i.e., for $a, b \in S$, the all terms in the OPE $a(z) b(w)$ can be expressed as normally ordered polynomials in the elements of $S$ and their derivatives. In the language of de Sole and Kac [DSK], the OPE algebra gives rise to a nonlinear conformal algebra satisfying skew-symmetry. There is a well-defined universal enveloping vertex algebra $\mathcal{U V}$ which is the initial object in the category of vertex algebras with the above strong generating set and OPE algebra. If for all fields $a, b, c \in S$ and integers $r, s \geq 0$, the Jacobi identities

$$
a_{(r)}\left(b_{(s)} c\right)-(-1)^{|a||b|} b_{(s)}\left(a_{(r)} c\right)-\sum_{i=0}^{r}\left(\begin{array}{l}
r \\
i
\end{array}\right)\left(a_{(i)} b\right)_{(r+s-i)} c=0,
$$

hold as formal consequences of the OPE relations, this Lie conformal algebra is then called a nonlinear Lie conformal algebra. The main result (Theorem 3.9) of [DSK] is that in this case, $\mathcal{U V}$ is freely generated by $S$. This means that it has a PBW basis consisting of monomials in the elements of $S$ and their derivatives.

In the examples $\mathcal{W}$ and $\mathcal{V}$ above, the universal enveloping vertex algebras are the universal $\mathcal{W}_{3}$-algebra with $c=-2$ and the universal $\mathcal{W}\left(\mathfrak{s p}_{4}, f_{\text {prin }}\right)$-algebra with $c=1$, respectively. Both of these are freely generated, so the associated varieties are isomorphic to $\mathbb{C}^{2}$ and the map (4.1) is an isomorphism in both cases. It is natural to ask whether (4.1) is always an isomorphism for universal enveloping vertex algebras, and in this section we provide a counterexample.

In [A], Adamovic studied a class of simple vertex algebra called $\mathcal{W}(2,2 p-1)$-algebras, where $p \geq 2$ is a positive integer. They are strongly generated by a Virasoro field $L$ with central charge $c=1-\frac{6(p-1)^{2}}{p}$, and a weight $2 p-1$ primary field $W$, and coincide with the singlet subalgebras of the $\mathcal{W}_{2, p}$-triplet algebras. The triplet algebras were the first examples of $C_{2}$-cofinite, nonrational vertex algebras to appear in the literature [AM].

We consider the case $p=3$, and we denote the $\mathcal{W}(2,5)$-algebra by $\mathcal{A}$. It can be realized explicitly inside the Heisenberg algebra $\mathcal{H}$ with generator $\alpha$ as follows.

$$
\begin{aligned}
L & =\frac{1}{2}: \alpha^{2}:+\sqrt{\frac{2}{3}} \partial \alpha \\
W & =\frac{1}{4 \sqrt{2}}: \alpha^{5}:+\frac{5}{4 \sqrt{3}}:(\partial \alpha) \alpha^{3}:+\frac{5}{12 \sqrt{2}}:\left(\partial^{2} \alpha\right) \alpha^{2}:+\frac{5}{8 \sqrt{2}}:(\partial \alpha)^{2} \alpha: \\
& +\frac{5}{48 \sqrt{3}}:\left(\partial^{3} \alpha\right) \alpha:+\frac{5}{24 \sqrt{3}}:\left(\partial^{2} \alpha\right) \partial \alpha:+\frac{1}{144 \sqrt{2}} \partial^{4} \alpha .
\end{aligned}
$$


The Virasoro field $L$ has central charge -7 , and the primary weight 5 field $W$ satisfies (7.3)

$$
\begin{aligned}
W(z) W(w) & \sim \frac{175}{12}(z-w)^{-10}-\frac{125}{6} L(w)(z-w)^{-8}-\frac{125}{12} \partial L(w)(z-w)^{-7} \\
& +\left(\frac{125}{3}: L L:-\frac{125}{8} \partial^{2} L\right)(w)(z-w)^{-6}+\left(\frac{125}{3}:(\partial L) L:-\frac{125}{36} \partial^{3} L\right)(w)(z-w)^{-5} \\
& +\left(50: L^{3}:+\frac{25}{24}:(\partial L)^{2}:-25:\left(\partial^{2} L\right) L:-\frac{175}{72} \partial^{4} L\right)(w)(z-w)^{-4} \\
& +\left(75:(\partial L) L^{2}:-\frac{175}{8}:\left(\partial^{2} L\right) \partial L:-\frac{125}{36}:\left(\partial^{3} L\right) L:-\frac{35}{96} \partial^{5} L\right)(w)(z-w)^{-3} \\
& +\left(\frac{25}{2}: L^{4}:+\frac{1175}{48}:(\partial L)^{2} L:+\frac{125}{12}:\left(\partial^{2} L\right) L^{2}:-\frac{775}{128}:\left(\partial^{2} L\right)^{2}:\right. \\
& \left.-\frac{225}{64}:\left(\partial^{3} L\right) \partial L:-\frac{175}{64}:\left(\partial^{4} L\right) L:-\frac{1115}{13824} \partial^{6} L\right)(z-w)^{-2} \\
& +\left(25:(\partial L) L^{3}:-\frac{25}{96}:(\partial L)^{3}:-\frac{125}{48}:\left(\partial^{2} L\right)(\partial L) L:+\frac{125}{24}:\left(\partial^{3} L\right) L^{2}:\right. \\
& \left.-\frac{775}{288}:\left(\partial^{3} L\right) \partial^{2} L:-\frac{425}{288}:\left(\partial^{4} L\right) \partial L:-\frac{115}{288}:\left(\partial^{5} L\right) L:-\frac{365}{24192} \partial^{7} L\right)(w)(z-w)^{-1}
\end{aligned}
$$

We have the following normally ordered relations in weights 8 and 10, respectively.

$$
\begin{gathered}
2: L \partial W:-5:(\partial L) W:-\frac{1}{6} \partial^{3} W=0 \\
: W^{2}:-: L^{5}:-\frac{335}{24}:(\partial L)^{2} L^{2}:-\frac{25}{3}:(\partial L) L^{3}:+\frac{283}{64}:\left(\partial^{2} L\right)(\partial L)^{2}: \\
+\frac{309}{64}:\left(\partial^{2} L\right)^{2} L:-\frac{67}{36}:\left(\partial^{3} L\right)(\partial L) L:+\frac{49}{216}:\left(\partial^{3} L\right)^{2}:-\frac{23}{32}:\left(\partial^{4} L\right) L^{2}: \\
+\frac{49}{64}:\left(\partial^{4} L\right)\left(\partial^{2} L\right):+\frac{249}{1280}:\left(\partial^{5} L\right) \partial L:+\frac{223}{3840}:\left(\partial^{6} L\right) L:+\frac{1}{504} \partial^{8} L=0 .
\end{gathered}
$$

It is straightforward to show using (7.4) and (7.5) that

$$
R_{\mathcal{A}} \cong \mathbb{C}[\ell, w] /\left\langle w^{2}-\ell^{5}\right\rangle .
$$

Here $\ell, w$ denote the images of $L, W$ in $R_{\mathcal{A}}$.

Next, let $\mathcal{U}=\mathcal{U} \mathcal{A}$ denote the universal enveloping vertex algebra of $\mathcal{A}$. By abuse of notation, we shall also denote the generators of $\mathcal{U}$ by $L, W$; they satisfy the same OPE relations as the generators of $\mathcal{A}$. We also denote by $\ell, w$ the images of $L, W$ in $R_{\mathcal{U}}$.

Lemma 7.1. $R_{\mathcal{U}} \cong \mathbb{C}[\ell, w] /\left\langle w^{2}-\ell^{5}\right\rangle \cong R_{\mathcal{A}}$.

Proof. Using (7.3), we can compute the left side of the Jacobi identity (7.1) in the case $a=b=c=W, r=4$ and $s=3$. We find that it does not vanish identically as a consequence of the OPE relations, but instead is given by

$$
\frac{9075}{16}\left(2: L \partial W:-5:(\partial L) W:-\frac{1}{6} \partial^{3} W\right) .
$$

Since all Jacobi identities must hold in any vertex algebra, (7.7) must be a null vector, so that (7.4) holds in $\mathcal{U}$. Therefore the corresponding Lie conformal algebra is not a nonlinear 
Lie conformal algebra, and $\mathcal{U}$ is not freely generated by $L$ and $W$. Applying the operator $W_{(2)}$ to the identity (7.4) yields a nonzero multiple of the identity (7.5). Therefore (7.5) also must hold in $\mathcal{U}$, which shows that the relation $w^{2}-\ell^{5}$ holds in $R_{\mathcal{U}}$. Since $R_{\mathcal{A}}$ is a quotient of $R_{\mathcal{U}}$, the claim follows.

Remark 7.2. We expect that $\mathcal{A}=\mathcal{U}$, but we do not prove this.

As in our previous example $\mathcal{W}$, even though the scheme $X_{\mathcal{U}}=\operatorname{Spec}\left(R_{\mathcal{U}}\right)$ is reduced, the arc space $\left(X_{\mathcal{U}}\right)_{\infty}$ is not. In particular,

$$
r=2 \ell^{(0)} w^{(1)}-5 \ell^{(1)} w^{(0)}
$$

is a nontrivial nilpotent element of $\mathcal{O}\left(\left(\tilde{X}_{\mathcal{U}}\right)_{\infty}\right)$ satisfying $r^{3}=0$, and $r \in \operatorname{ker}\left(\Phi_{\mathcal{U}}\right)$. Therefore $\mathcal{U}$ is an example of a universal enveloping vertex algebra for which the map (4.1) fails to be injective.

Finally, via the embedding

$$
\mathcal{H} \rightarrow \mathcal{S}, \quad \alpha \mapsto \sqrt{-1}: \beta \gamma:
$$

$\mathcal{A}$ can be identified with a subalgebra of $\mathcal{S}$. By the same argument as Theorem 5.2, one can check that the map on varieties induced by (4.1) is an isomorphism. Therefore the same holds for $\mathcal{U}$.

\section{REFERENCES}

[A] D. Adamovic, Classification of irreducible modules of certain subalgebras of free boson vertex algebra, Journal of Algebra 270 (2003) 115-132.

[AM] D. Adamovic and A. Milas, On the triplet vertex algebra $\mathcal{W}(p)$, Adv. Math. 217 (2008), no. 6, 26642699.

[ArI] T. Arakawa, A remark on the $C_{2}$ cofiniteness condition on vertex algebras, Math. Z. 270 (2012), no. 1-2, 559-575.

[ArII] T. Arakawa, Associated Varieties and Higgs Branches (A Survey), Contemp. Math. 711(2018), 37-44.

[ArIII] T. Arakawa, Representation theory of W-algebras and Higgs branch conjecture, Proc. Int. Cong. of Math 2018 Rio de Janeiro, Vol. 1 (1261-1278).

[AK] T. Arakawa and K. Kawasetsu, Quasi-lisse vertex algebras and modular linear differential equations, arXiv:1610.05865 [math.QA], to appear in Kostant Memorial Volume, Birkhauser.

[AMI] T. Arakawa and A. Moreau, Lectures on $\mathcal{W}$-algebras, preprint.

[AMII] T. Arakawa and A. Moreau, Arc spaces and chiral symplectic cores, arXiv:1802.06533, to appear in the special issue of Publ. Res. Inst. Math. in honor of Professor Masaki Kashiwara's 70th birthday.

[BLL+] C. Beem, M. Lemos, P. Liendo, W. Peelaers, L. Rastelli, and B. C. van Rees, Infinite chiral symmetry in four dimensions, Comm. Math. Phys., 336(3):1359-1433, 2015.

[BR] C. Beem and L. Rastelli, Vertex operator algebras, Higgs branches, and modular differential equations, J. High Energ. Phys. (2018) 2018: 114. https:/ /doi.org/10.1007/JHEP08(2018)114.

[B] R. Borcherds, Vertex operator algebras, Kac-Moody algebras and the monster, Proc. Nat. Acad. Sci. USA 83 (1986) 3068-3071.

[BS] D. Bourqui and J. Sebag, The radical of the differential ideal generated by $X Y$ in the ring of two variable differential polynomials is not differentially finitely generated, to appear Journal of Comm. Algebra (2017).

[DSK] A. de Sole and V. Kac, Freely generated vertex algebras and non-linear Lie conformal algebras, Comm. Math. Phys. 254 (2005), no. 3, 659-694.

[DN] C. Dong and K. Nagatomo, Classification of irreducible modules for the vertex operator algebra $M(1)^{+}$, J. Algebra 216 (1999), no. 1, 384-404.

[EM] L. Ein and M. Mustata, Jet schemes and singularities, Algebraic geometry-Seattle 2005. Part 2, 505546, Proc. Sympos. Pure Math., 80, Part 2, Amer. Math. Soc., Providence, RI, 2009. 
[EH] J. van Ekeren and R. Heluani, Chiral homology of elliptic curves and Zhu's algebra, arXiv:1804.00017 [math.QA].

[FBZ] E. Frenkel and D. Ben-Zvi, Vertex Algebras and Algebraic Curves, Math. Surveys and Monographs, Vol. 88, American Math. Soc., 2001.

[FLM] I. B. Frenkel, J. Lepowsky, and A. Meurman, Vertex Operator Algebras and the Monster, Academic Press, New York, 1988.

[K] V. Kac, Vertex Algebras for Beginners, University Lecture Series, Vol. 10. American Math. Soc., 1998

[KS] K. Kpognon, J. Sebag, Nilpotency in arc schemes of plane curves, Comm. in Algebra, Vol. 45 no 5 (2017), 2195-2221

[Kol] E. Kolchin, Differential algebra and algebraic groups, Academic Press, New York 1973.

[LiI] H. Li, Vertex algebras and vertex Poisson algebras, Commun. Contemp. Math. 6 (2004) 61-110.

[Mi] M. Miyamoto, Modular invariance of vertex operator algebras satisfying C2-cofiniteness. Duke Math. J. 122(1), 51-91 (2004).

[SI] J. Sebag, Arcs schemes, derivations and Lipman's theorem, J. Algebra 347 (2011) 173-183.

[SII] J. Sebag, A remark on Berger's conjecture, Kolchin's theorem and arc schemes, Archiv der Math., Vol. 108 no (2017), 145-150

[WaI] W. Wang, $\mathcal{W}_{1+\infty}$ algebra, $\mathcal{W}_{3}$ algebra, and Friedan-Martinec-Shenker bosonization, Comm. Math. Phys. 195 (1998), no. 1, 95-111.

[WaII] W. Wang, Classification of irreducible modules of $\mathcal{W}_{3}$ algebra with $c=-2$, Comm. Math. Phys. 195 (1998), no. 1, 113-128.

[Za] A.B. Zamolodchikov, Infinite extra symmetries in two-dimensional conformal quantum field theory (Russian), Teoret. Mat. Fiz. 65 (1985), 347-359. English translation, Theoret. and Math. Phys. 65 (1985), 1205-1213.

[Zh] Y. Zhu, Modular invariants of characters of vertex operators, J. Amer. Soc. 9 (1996) 237-302.

Research INSTITUTE FOR MATHEMATICAL SCIENCES, KyOto UNIVERSity

E-mail address: arakawa@kurims .kyoto-u.ac.jp

UNIVERSITY OF DENVER

E-mail address: andrew.linshaw@du.edu 